\title{
OVERVIEW AND PERFORMANCE OF THE UKRAINIAN SLR STATION “LVIV-1831”
}

\author{
Ja. Blagodyr, A. Bilinsky, K. Martynyuk-Lototsky, O. Lohvynenko, N. Virun \\ Astronomical Observatory of Ivan Franko National University of Lviv, \\ Ukraine, 79005, Lviv, Kiril and Mephodij St.8 \\ e-mails: blagod@astro.franko.lviv.ua, slr1831@ukr.net
}

\begin{abstract}
The paper presents the current status and performance of the SLR station "Lviv-1831". The present equipment allows ranging of satellite not lower than $900 \mathrm{~km}$ with a single shot precision about $50 \mathrm{~mm}$. The team works over system upgrade and performance improvement. It includes: neutral filters wheel developing for equilibration of the amplitude of reflected pulses from different satellites, building up the shutter in the face of PMT for the spurious light pulses blocking. Also the new adjusting of the telescope optical mechanical systems was made. After that investigation the new telescope error model was built with an accuracy 20arcsec that allowed at the first time test ranging of the "shadowed" satellite passes. In the near future the team will finish installation and testing of the new PMT Hamamatsu H6780. Maiden upgrades should better the performance and ranging accuracy by the factor of 2-3.
\end{abstract}

Keywords: satellite laser ranging, slr, ILRS, ITRF

\section{INTRODUCTION AND GENERAL CHARACHTERISTICS}

Satellite laser ranging station "Lviv-1831" was found in 1998. In August 2002, it was registered as associate SLR station in ILRS (Pearlman et al., 2002), SOD number 18318501, DOMES 12368S001. Also, it is a member of the Ukrainian network of UCEOP (Ukrainian Center for Earth Orientation Parameters) (UCEOP).

The station (fig.1) is based on the next equipments:

1. $1 \mathrm{~m}$ telescope TPL-1M on alt-azimuth mounting

a. maximum tracking speed $2 \%$ sec

b. tracking accuracy is about 25arcsec - that big value is connected with the lack of encoders. Tracking system works by means of stepper motors pulses counting. The optical-mechanical errors of the telescope are taken into account by the observing of reference stars and building of the corresponding model. And that's why all observations are conducted in most cases with the visual guiding by observer.

c. horizon mask angle $20^{\circ}$ 
2. Laser transmitter SL-212

a. wavelength $532 \mathrm{~nm}$, pulse energy $60 \mathrm{~mJ}$, pulse width $150 \mathrm{ps}$, repetition rate $5 \mathrm{~Hz}$, output beam divergence $10^{\prime \prime}$.

3. Receiving system

a. Start - photodiode LF-2, discriminator ORTEC935 (200MHz constant fraction discriminator with a walk 25ps over 100:1 dynamic range).

b. Stop - soviet PMT FEU-136 (rise time 2ns), pulse amplifier VT120, discriminator ORTEC935

4. Registration system - computerized timer A911 with internal accuracy 40 ps.

5. Time and frequency service

a. Rubidium standard SChV74 - stability $10^{-11}$, time synchronization accuracy 1usec

b. GPS receiver - Motorola UTOncore - 1pps accuracy 50nsec

6. Meteostation WS3600

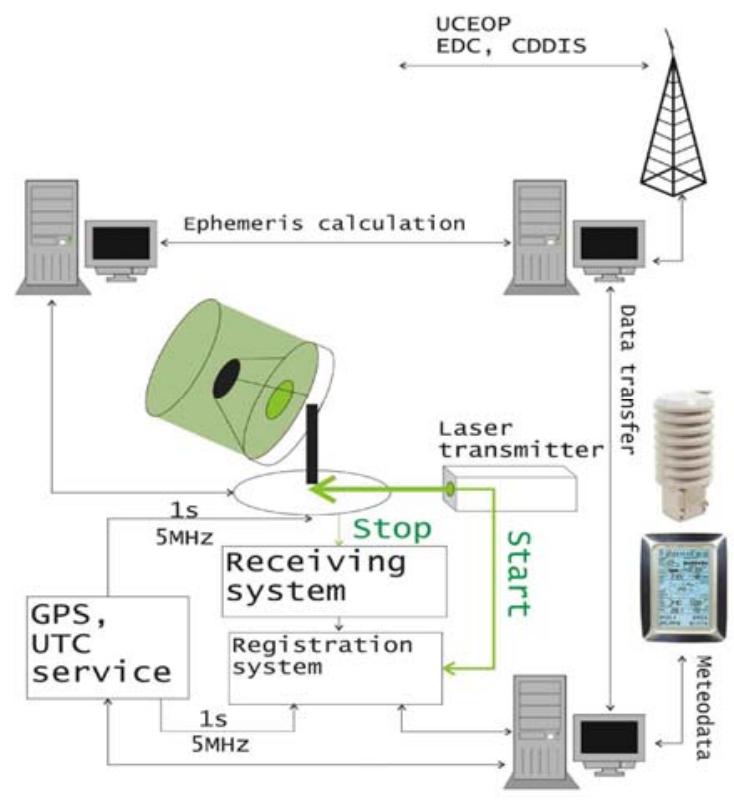

Fig.1. Scheme of the SLR station "Lviv-1831”.

The block of receiving and processing the data integrates three program systems - Comtis, SatComp and WinOncore. Program system Comtis is providing mode control of computerized Timer of Events A911(worked out in Latvian university). Program system SatComp, which was developed in Latvian university, is necessary for preliminary data processing of satellite laser ranging observations. Program complex WinOncore reply for work control of GPS-receiver UTOncore. All programs work under MS Windows98.

Current telescope control software is also developed in Latvian University and works under MSDOS.

The current fire-receiving system allows of only above $900 \mathrm{~km}$ ranging (Bilinsky et al., 2005). This limit is appeared because of the system construction. It consists of two rotating mirrors with diameters about $80 \mathrm{~mm}$ that rotate with the frequency $10 \mathrm{~Hz}$. And that's why the laser (upper) mirror can clear the optical path for the receiving mirror only in the period not less than $6 \mathrm{~ms}$ that corresponds to range of $900 \mathrm{~km}$. 


\section{STATION PERFORMANCE}

During 2005 on the station 138 passes of LAGEOS satellites were tracked with single shot RMS 50mm. The short term stability over 2005 is about $35 \mathrm{~mm}$, and the long term $-25 \mathrm{~mm}$ (fig.3) (ILRS Report). On fig.3 the lines on the level $10 \mathrm{~mm}$ and $20 \mathrm{~mm}$ are the ILRS require-

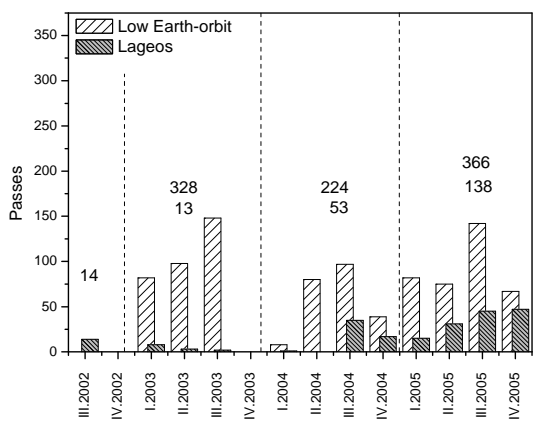

Fig.2a. Tracked passes statistics.

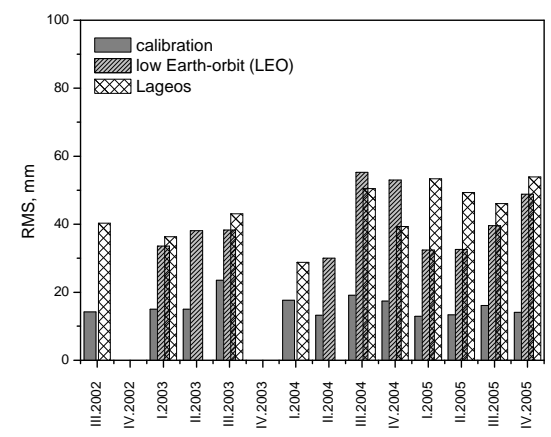

Fig.2b. RMS of the SLR results (ILRS Report)

ments to the NP RMS and results stability correspondingly. The short term stability (as is used by ILRS) is the measure of short term bias stability, in millimeters, during the last quarter. The short term stability is computed as the standard deviation about the mean of the pass-by-pass range biases (minimum number of passes in quarter is 10). The long term stability (as is used by ILRS) is the measure of long term bias stability, in millimeter, during the past year. The long term stability is the standard deviation of the monthly range bias estimates. A station must have tracked LAGEOS $(1,2)$ in at least 8 of the last 12 months in order to compute this metric.

The small amount of SLR observations (fig.2a), especially LEO, is concerned with the fact that we can observe only the objects illuminated by Sun. The main problems in the tracking of "shadowed" objects are initial acquiring and further guiding. As we haven't encoders on the telescope, the only way to take into account mounting and optical errors is the

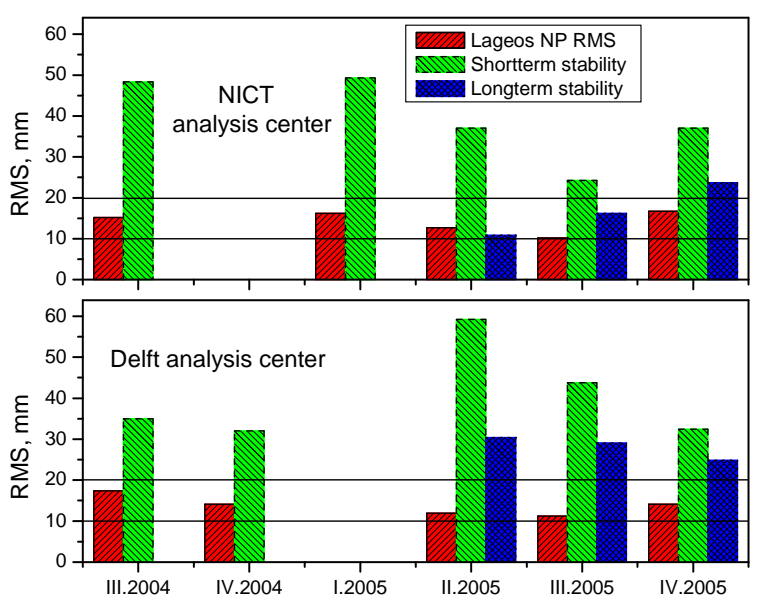

Fig.3. Stability of the SLR results (ILRS Report).

telescope model. In 2006 we again made global investigation and adjusting of the telescope. Its included: checking the horizontality of the telescope platform, investigation of the azimuth pivoting dolly raceway, adjusting of all flat mirrors, refocusing of the telescope on 532nm. 
These researches showed that the vertical axis of the telescope strongly depend on azimuth angle of the telescope pointing. Formerly we assumed that it had been independent of azimuth. Then several series of stars observations were done. On the basis of these results the new model of telescope was made. The accuracy of the new model is 20arcsec in contrast to the previous one with 120arcsec accuracy.

In October 2006 the first successful SLR observations of "shadowed" satellite were conducted:

- October 9, 0:35UTC Ajisai satellite, 981 (20 normal) points were obtained (324 (9 normal) points were obtained in the shadow), rms 44mm;

- October 13, 20:04UTC Ajisai satellite, 620 (16 normal) points - 413 (12 normal) in shadow, rms $48 \mathrm{~mm}$.

These observations were actually testing "shadowed" ones, and further we would continue them.

\section{CURRENT AND FUTURE HARDWARE UPGRADES}

The first system upgrade since ILRS network inclusion was made in 2005. The new meteostation WS3600 was purchased and installed on the station (fig.1). It has the next parameters: temperature accuracy $0.1^{\circ} \mathrm{C}$, pressure accuracy 0.3 mbar, humidity accuracy $4 \%$.

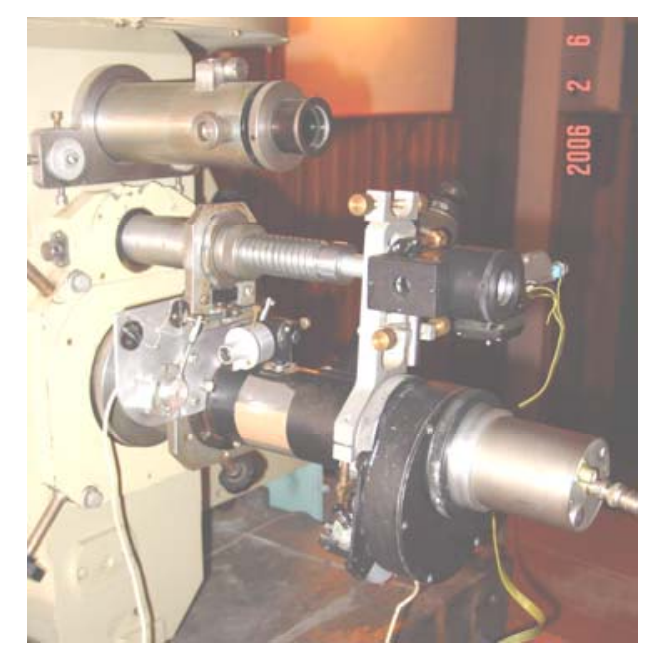

Fig.4a. Receiver part modernization: filters Fig.4b. Additional TV guiding system. wheel, rotating shutter, new PMT module.

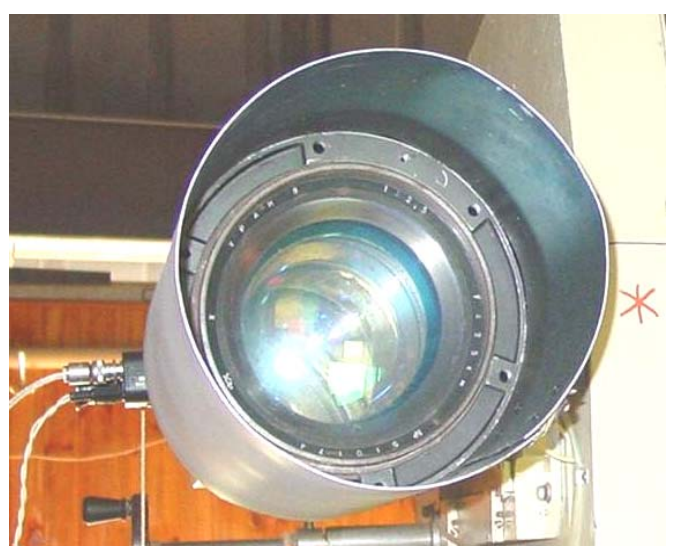

At present, the station team works over testing of:

- new PMT receiver - Hamamatsu module H6780-20

0 rise time $0.78 \mathrm{~ns}$

o peak sensitivity wavelength of module $\mathrm{H} 6780-20$ is $500-630 \mathrm{~nm}$

o minimum luminous cathode sensitivity $-350 \mu \mathrm{A} / \mathrm{lm}$

o minimum luminous anode sensitivity - $35 \mathrm{~A} / \mathrm{lm}$

o noise free gain of 500,000 times

- neutral filters wheel for light pulses power reductions

- new electromechanical shutter (fig.4a).

Implementing these improvements in the system of SLR station "Lviv-1831" can increase the performance and the accuracy of ranging results by a factor of about two to three. The electromechanical modulator was installed in the receiving channel of the telescope for background laser flashes reducing. In the moment of start laser pulse the modulator shutters 
the PMT cathode. Using of the neutral filters wheel should extend indirectly the dynamic range of the discriminator and thus ensure more accurate observations of all satellite types with different signal response.

For a better satellite acquisition and other positioning astrometry tasks, the new guiding system was installed on the telescope TPL. The system consists of the objective-refractor Uran-9 ( $D=100 \mathrm{~mm}, F=250 \mathrm{~mm})$ equipped with sensitive TV CCD camera LCL902K (sensitivity is 0.00015lux, $1 / 2$ inch CCD matrix) (fig.4b). Field of view is 64arcmin $x$ $86 a r c m i n$. This equipment allows us to observe objects up to 12 mag with 10 s storage.

The next step in station modernization is the altering of firing-receiving obturator for ranging of very low satellites at altitudes about $500 \mathrm{~km}$ and timing system upgrading.

\section{SOFTWARE UPDATES}

The Lviv SLR station software consist of:

- Telescope tracking software maiden in Riga university operates under MSDOS. PC is a Pentium 200MHz computer.

- Registration, preprocessing and timing service software operates under Windows98. $\mathrm{PC}$ is a Celeron $400 \mathrm{MHz}$ computer.

o Comtis - registration software maiden in Riga University. It controls the event timer A911 providing calibration and tracking modes, window width changing in the wide range. The start window width is 20000ns and after satellite capturing one can narrow it to 1 ns to reduce false pulse counting. Typically we work with window width about 200ns.

- SLR data communication is provided through radio Internet $128 \mathrm{kps}$ connection by PC AMD64 3000MHz that operates under Linux.

The current SLR tracking and receiving software was upgraded for the new initial data format, i.e. CPF predictions.

Also the station team works over new tracking software under operating system RealTime Linux v.3.1 (Bilinsky and Melekh, 2005). The new software should allow us to optimize and automate the SLR observations. Currently we continue real-time module development and testing, including telescope control algorithm development (optimization of methods for better target acquiring and tracking).

\section{ITRF POSITION COORDINATES}

As seen from the fig.2a, the SLR station "Lviv-1831" began the observations only in 2002 (that were sent to EUROLAS Data Center). And only in the end of 2004 we began LAGEOS ranging intensively enough to make some analysis (fig.3).

For the period 2002-2003 the obtained coordinates are in general the estimations but not the solutions. On the basis of their analysis, it can be said that on the level $0.170 \mathrm{~m}$ there are no systematic biases and trends in the SLR results of the station "Lviv-1831" in the searched period.

During year 2005, on the SLR station “Lviv-1831" there were 138 LAGEOS observations conducted with the single shot RMS about $45 \mathrm{~mm}$ (15mm on normal points) (figs.2a,2b,3). ITRF2005 solution of 12368 S001 (SLR 1831) at the epoch 2000.0 (ITRF): 


$$
\begin{array}{ll}
X=3760674.585 \pm 0.076 \mathrm{~m} & \mathrm{Vx}=31.2 \pm 14.2 \mathrm{~mm} / \text { year } \\
Y=1670776.413 \pm 0.068 \mathrm{~m} & \mathrm{Vy}=4.4 \pm 12.9 \mathrm{~mm} / \text { year } \\
\mathrm{Z}=4857165.267 \pm 0.062 \mathrm{~m} & \mathrm{Vz}=34.8 \pm 11.4 \mathrm{~mm} / \text { year }
\end{array}
$$

\section{CONCLUSIONS}

Currently on the station we can observe about 30\% of passes number required by ILRS with an accuracy corresponding to upper level of the mentioned above requirements. These things will be improved by means of maiden on the station hardware upgrades:

- The new PMT was purchased with better characteristics (rise time 0.78ns etc.). After final testing of the new PMT module H6780-20 we hope to improve the SLR accuracy by a factor of 2-3.

- The neutral filters wheel was developed, produced and installed in Lviv station, that give us an opportunity to receive the same amplitude reflected signal from different satellites, that allows to increase the accuracy of obtained data.

- The shutter was developed and built up, which protect the PMT channel from scattered radiation at the time of laser transmitter shots.

- A new research of telescope optical mechanical errors was conducted. This allowed us to calculate a new telescope errors model by the star observations. The mean accuracy of the new model is 20arcsec in contrast with the previous one that has 120arcsec mean accuracy. This model improvement gave us opportunity at first to observe "shadowed" satellite passes.

The works presented in the first three points are conducted now in the testing mode and require further research. The last one (telescope errors research) is completed at this stage. Now telescope pointing to begin of a satellite path is without 25arcsec at most cases. For "shadowed" passes observations we can increase it by observation of nearby star and corresponding correction of the telescope errors model. This method allows us to achieve a pointing in the begin with accuracy about 10 arcsec which is a maximum for our system. That value is compared with laser beam divergence and as the testing observations has show is enough for ranging without visual guiding. We will continue to observe "shadowed" satellites to gain more experience in such observations.

The next steps in the station upgrading are: to conduct a testing observations with the new PMT and compare with the old one; to change firing-receiving system fundamentally to reject as much as possible moving parts of the system to get an ability of ranging very low satellites; to purchase and install a new modern event timer with better operating characteristics.

Acknowledgements. The installing of the stable high-speed Internet channel was funded by INTAS grant \#03-59-11. Also we are grateful to ILRS and UCEOP for their informational support on SLR results data processing and analysis.

\section{REFERENCES}

Bilinsky A., Blagodyr Ya., Lohvynenko A. and Ternavska S. Station reports: Lviv, Ukraine, International laser Ranging Service 2003-2004 Annual Report, pp.B-26 - B-27, June 2005.

Bilinsky A. and Melekh B. Control of the laser ranger TPL-1M in RTLinux, Problems of the control and informatics, No.2 (2005), pp.103-106 (in Russian).

Pearlman, M.R., Degnan, J.J., and Bosworth, J.M., "The International Laser Ranging Service", Advances in Space Research, Vol. 30, No. 2, pp. 135-143, July 2002. 
ILRS Report SLR Global Performance Report Card

http://ilrs.gsfc.nasa.gov/stations/site_info/global_report_cards/index.html

ITRF web site http://itrf.ensg.ign.fr/

UCEOP - Ukrainian Center for Earth Orientation Parameters

http://www.mao.kiev.ua/EOP/ENGLISH/structure.htm

Received: 2006-12-18,

Reviewed: 2007-05-02,

Accepted: 2007-10-01. 